\title{
SCADidoC
}

Infer, Interpret \& Inspire Science

International Journal of Dentistry and Oral Science (IJDOS)

ISSN: 2377-8075

\section{Translational Oral Science Implications for Systemic Clinical Research}

Chiappelli $\mathrm{F}^{1,23^{*}}$

${ }^{1}$ Oral Biology \& Medicine, Center for the Health Sciences, UCLA, USA.

${ }^{2}$ Health Sciences, California State University, Northridge, USA.

${ }^{3}$ Evidence-Based Decisions Practice-Based Research Network.

\section{Abstract}

Modern contemporary oral science brings forward timely and critical contributions to translational science, the convergence of translational research and translational effectiveness. Incontrovertible evidence has established oral fluids biomarkers as reliable and replicable materials for assessing, for example, cortisol levels following challenges of the hypothalamic pituitary adrenal (HPA) axis, such as is the case of experimental stress challenges (e.g., Trier stress test), for monitoring cortisol resistance in certain patient populations, as well as for assessing cytokine levels as markers of cell-mediated immunity (CMI) activation, because HPA and CMI activation usually cross-regulate and interact. Equally as important in our global warming environment and consequential advancing tropical diseases, oral science critically informs translational research in patients afflicted with Zika, Dengue and related viruses.

Keywords: Saliva; Hypothalamic Pituitary Adrenal Axis (HPA); Cell Mediated Immunity (CMI); Trier Stress Test; Personenvironment Fit; Cortisol; Cytokines; Human Immunodeficiency Virus (HIV); Zika Virus; Comparative Effectiveness Research (CER).

\section{Introduction}

There is incontrovertible evidence supporting the use of oral fluids (e.g., whole saliva, parotid saliva, crevicular fluid) in translational research. From the early assessment of cortisol and other hormones to the characterization of the circadian variation of pro-inflammatory, TH1 and TH2 cytokines, to the now well-established recognition of molecular and viral biomarkers in oral fluids, oral science has acquired significant importance as a source of physiologically relevant and noninvasive samples for monitoring systemic responses across a wide spectrum of clinical research [1-4].

Case in point, salivary cortisol is now one of the preferred measures in investigations of the resilience of the hypothalamic-pituitary-adrenal (HPA) axis in patients with psychiatric diseases, as well as in normal healthy individuals subjected to experimental stress paradigms [5-8]. Cell-mediated immunity (CMI) responses are also reliably measured in saliva and other oral fluids (e.g., crev- icular fluids), and consistently occur in parallel to HPA activation in healthy control subjects. Salivary cytokines are now established as reliable biomarkers of a variety of oral conditions, from periodontal disease [9] to candida-mediated oral pathologies [10], as well as of systemic CMI responses, from cancer [11] to stress [12].

Translational research, defined by the National Institutes of Health $(\mathrm{NIH})$, is directed at obtaining biopsy samples from patients, defining and characterizing the cellular and the molecular characteristics of the pathology in the laboratory to then integrate this information in better patient-centered clinical decision-making (from the patient/bed/dental chair - to the laboratory bench - back to the patient/bed/dental chair). Translational effectiveness, defined by the Agency of Healthcare Research and Quality (AHRQ), arises from a structured and detailed patient-clinician encounter to engender a systematic process of patient-centered outcomes research and research synthesis that lead to the appropriate translation of comparative effectiveness findings into specific evidence-based intervention recommendations for practical

*Corresponding Author:

Francesco Chiappelli Ph.D., Dr. Endo (h.c.),

Professor, UCLA School of Dentistry, USA.

Tel: +13107946625

Fax: 310-794-7109

Email: fchiappelli@dentistry.ucla.edu

Received: February 11, 2016

Accepted: March 07, 2016

Published: March 09, 2016

Citation: Chiappelli F (2016) Translational Oral Science Implications for Systemic Clinical Research. Int J Dentistry Oral Sci. 03(3), 209-212. doi: http://dx.doi.org/10.19070/2377-8075-1600044

Copyright: Chiappelli $\mathbf{F}^{\circ}$ 2016. This is an open-access article distributed under the terms of the Creative Commons Attribution License, which permits unrestricted use, distribution and reproduction in any medium, provided the original author and source are credited. 
use in evidence-based, effectiveness-focused and patient-centered clinical decision-making. Translational science in health in general, and in dentistry in particular positions itself at the very convergence of efficacy-driven translational research and effectivenessfueled translational effectiveness $[13,14]$.

\section{Translational Oral Science}

We previously discussed the critical role of testing biomarkers in saliva, among other oral fluids and in other body fluids, for crafting and testing new hypotheses in diagnosis and patient-centered treatment interventions for patients with HIV/AIDS [15], Dengue [16], Ebola [17], and other viral infections, including Zika [18] that trigger the onset of HPA-CMI activation, and which are current important public health threats. In that context, we characterized the circadian variation of diverse pro-inflammatory and TH1 and TH2 cytokines in saliva to demonstrate to potential use of oral fluids in psychobiology research. We proffered as an example the Trier psycho-socio/cognitive situational stress (mental arithmetic task and free speech in front of an audience), which remains to this day one among the most useful, reliable human stress models, because it yields comparative data of the psychobiology of stress, including its measurement by salivary amylase $[19,20]$.

In brief, the Trier Social Stress Test (TSST) is a laboratory experimental protocol designed to induce replicable psycho-social stress in human subjects [21]. The test presents stress challenges in a rigorous and standardized format that combines different stressgenerating tasks, including elements of public speaking, mental arithmetic, and anticipation, to induce a remarkable large physiological response that comprises significant elevations in salivary cortisol about $10 \mathrm{~min}$ following the conclusion of the stress protocol in most normal health subjects. Stress-related free salivary cortisol and total plasma cortisol net increases do not differ as a function of the time of day (morning or afternoon) when the Trier test is administered, despite the fact that morning vs. afternoon pre-stress salivary and plasma cortisol levels differ significantly due to diurnal variations [22].

Males generally respond with a more vigorous elevation in cortisol across all body fluids, compared to pre-menopausal females, in part because of the differential confounding effect of female hormones at different stages of the menstrual cycle [23]. Across genders, smoking (i.e., nicotine) and drinking (i.e., alcohol) blunt the psychobiological response to the Trier stress test. By contrast, certain conditions, which are known to modulate HPA responsiveness, such as Major Depression [24], significantly enhance the psychobiological response to the Trier stress.

Patients with temporomandibular disorders (TMD) also show a significantly higher cortisol response to the experimental Trier stress test, compared to age-and sex-matched control subjects. Indeed, the power of the Trier test, combined with the replicability of salivary cortisol measurements revealed that TMD patients are heterogeneous with respect to the HPA axis response to stress. One group of patients with TMD are hyper-secretors of cortisol following the Trier test, another group exhibits rises in salivary cortisol post-stress that are not significantly different from control subjects. That second group of TMD patients typically shows a significant negative relationship between salivary cortisol response to the Trier test and self-reported symptoms of anxiety or depression. Taken together these translational research observations provide timely and critical patient-centered information regarding physiologically important differences among subgroups of patients with TMD in terms of HPA-CMI interactions and its relation to anxiety [25].

We proposed elsewhere that TMD and its sequelae may be more prevalent than actually recorded in medical histories among patients with HIV/AIDS in part because of their related "fragile bone syndrome" and underlying osteoimmunopathology. We inferred that the probability that patients with HIV/AIDS may suffer from TMD with a prevalence significantly greater than epidemiological statistics (i.e., $>75 \%$ ) suggest is clinically relevant. We also surmised that, since in many patients with TMD, the auriculotemporal nerve, a terminal nerve ending of the 2 nd branch of the trigeminal cranial nerve (CV-II, maxillary branch), may be compressed, irritated, or otherwise damaged, there may be important central nervous secondary involvement in TMD [26], which could mask the traditional epigenetic and immunopathological biomarkers of emerging neuroAIDS [27].

Certainly, the Trier test is not the only means of reliably assessing psycho-emotional stress and adaptation. The person-environment fit (PE-fit) theoretical model, although not a stress challenge as the Trier test, is an approach to quantifying such analyses in the pursuit of patient-targeted interventions. We have described the value of the P-E fit model in dental practice both in terms of the dental needs of patients with senile dementia of the Alzheimer's type [28], and are beginning to validate in our ongoing studies of dental patients in general [29] and patients with TMD specifically [30].

In brief, the P-E fit model seeks to identify the key points of the subject subjective perception and objective reality of fit to the demands of the surrounding environment. In concert with translational effectiveness, it aims at preventive intervention to improve the person's reality contact, quality of life and overall well-being. The model acknowledges that, to a large extent, the perception of fit of an individual within the environment is dependent upon one's ability to discern the distinction vs. overlap between perception and reality. It posits that a misfit between the person and environment leads to seriously impaired outcomes of life satisfaction and performance [28-30].

\section{The Relevance of Translational Oral Science in the Next Decades}

A recently emerging literature documents the "oral virome", which refers to the clinically important novel branch of oral science that pertains to the survey and characterization of viral infections in saliva and other oral fluids. Case in point, norovirus, rabies, human papillomavirus, Epstein-Barr virus, herpes simplex viruses, hepatitis $\mathrm{C}$ virus, and HIV that are commonly detected in salivary samples. Increasingly, viral infections can be diagnosed using a oral biopsy samples, such as saliva, mucosal transudate, oral swab, etc. These samples are less invasive to obtain compared to blood or cerebro-spinal fluid, and typically more stable than urine [31].

In that context, we have recently proposed that the same HPA- 
CMI interactions that underlie the psychobiological response to stress (e.g., Trier test) also drive the underlying physiopathology observed in patients with HIV/AIDS. Several among these patients, when treated with anti-retroviral therapy, manifest an immune reconstitution inflammatory syndrome that is suggestive of deregulated HPA-CMI, and which often manifests as heightened anxiety quantifiable by the P-E fit model. Biomarkers in saliva and other oral fluids will be critical in testing whether or not this pathological profile can be attributed in systemic glucocorticoid resistance that sub-group of HIV/AIDS patient [18].

Furthermore, as we now experience global climate change and associated warming of the northern hemisphere, Western societies are experiencing a novel threat by a plethora of tropical diseases. It is possible and even probable that oral fluids may take an increasingly new and important role in yielding key diagnostic and prognostic biomarkers. Case in point, viral infections and other challenges to immune surveillance yield both a CMI response - of course - and an associated HPA response. Both systems are stimulated, and yield elevated levels of cortisol and of pro-inflammatory cytokines in all body fluids, including oral fluids.

We have proposed that deregulated HPA-CMI responses, detectable in oral fluids, may play an important role in the pathological mechanisms that underlie Dengue [16] and Zika virus infections [18]. Both viruses are members of the genus Flavivirus, which also includes the West Nile virus, the tick-borne encephalitis virus, the Chikungunya virus, the Yellow Fever virus. Flaviviruses are enveloped positive-sense single-stranded RNA viruses, transmitted to humans by means of a bite of the infected mosquito of the genus Aedes (i.e., A. albopictus, A. aegypti). The Zika virus (ZIKV) can be detected in salivary samples: in fact, the commercially available specific ZIKV RT-PCR, validated for specificity and sensitivity, is reported to detect ZIKV more reliably and with higher precision, and earlier following infection in saliva, compared to blood, plasma or serum [32].

\section{Conclusion}

Taken together, the novel lines of evidence discussed here emphasize the timely and critical importance of oral science in the larger context of translational research and translational effectiveness. Translational oral science will increasingly integrate patienttargeted biological discoveries - that is, translational research proffered by biomarkers obtained from oral fluids - with effective patient-centered interventions (i.e., translational effectiveness). The next decades will establish oral science as a premier driver of research on neuroendocrine-immune interactions in normal healthy individuals, in patients traditionally cared for in the dental practice (e.g., patients with TMD, or with HIV/AIDS oral manifestations), and in patients afflicted with novel, emerging or tropical diseases, such as infections with the Dengue or the Zika virus.

\section{Acknowledgements}

The author thanks the Evidence-Based Decisions Active Groups of Stakeholders (EBD-AGS) of the EBD-Practice-Based Research Network (ebd-pbrn.org), and the students and colleagues of the EBD Study Group, for edifying discussions. Funded in part by UCLA Senate grants and Fulbright Specialist grant (5077) to FC.

\section{References}

[1]. Bosch JA (2014) The use of saliva markers in psychobiology: mechanisms and methods. Monogr Oral Sci 24: 99-108.

[2]. Bonne NJ, Wong DT (2012) Salivary biomarker development using genomic, proteomic and metabolomic approaches. Genome Med 4(10): 82.

[3]. Nunes LA, Mussavira S, Bindhu OS (2015) Clinical and diagnostic utility of saliva as a non-invasive diagnostic fluid: a systematic review. Biochem Med 25(2): 177-192.

[4]. Zhang Y, Sun J, Lin CC, Abemayor E, Wang MB, et al. (2016) The emerging landscape of salivary diagnostics.Periodontol 70(1): 38-52.

[5]. Cevada T, Vasques PE, Moraes H, Deslandes A (2014) Salivary cortisol levels in athletes and nonathletes: a systematic review. Horm Metab Res 46(13): 905-910.

[6]. Hayes LD, Grace FM, Baker JS, Sculthorpe N (2015) Exercise-induced responses in salivary testosterone, cortisol, and their ratios in men: a metaanalysis. Sports Med 45(5): 713-726.

[7]. Herane Vives A, De Angel V, Papadopoulos A, Strawbridge R, Wise T, et al. (2015) The relationship between cortisol, stress and psychiatric illness: New insights using hair analysis. J Psychiatr Res 70: 38-49.

[8]. Jakuszkowiak-Wojten K, Landowski J, Wiglusz MS, Cubała WJ (2015) Cortisol as an indicator of hypothalmic-pitituary-adrenal axis dysregulation in patients with panic disorder: a literature review. Psychiatr Danub 27(Suppl 1): S445-S451.

[9]. Jaedicke KM, Preshaw PM, Taylor JJ (2016) Salivary cytokines as biomarkers of periodontal diseases. Periodontol 2000 70(1): 164-183.

[10]. Salvatori O, Puri S, Tati S, Edgerton M (2016) Innate Immunity and Saliva in Candida albicans-mediated Oral Diseases. J Dent Res.

[11]. Idris A, Ghazali NB, Koh D (2015) Interleukin 1 $\beta$-A Potential Salivary Biomarker for Cancer Progression? Biomark Cancer 7: 25-29.

[12]. Slavish DC, Graham-Engeland JE, Smyth JM, Engeland CG (2015) Salivary markers of inflammation in response to acute stress. Brain Behav Immun 44: 253-269.

[13]. Chiappelli F (2014) Fundamentals of Evidence-based Health Care and Translational Science. Springer-Verlag, Heidelberg.

[14]. Chiappelli F (2015) Comparative Effectiveness Research (CER): New Methods, Challenges and Health Implications. Nova Science Publishers, New York.

[15]. Orlando B, Giacomelli L, Chiappelli F, Barkhordarian A (2013) New tools for the investigations of Neuro-AIDS at a molecular level: The potential role of data-mining. Bioinformation 9(12): 656-658.

[16]. Chiappelli F, Santos SM, Caldeira Brant XM, Bakhordarian A, Thames AD, et al. (2014) Viral immune evasion in dengue: toward evidence-based revisions of clinical practice guidelines. Bioinformation 10(12): 726-733.

[17]. Chiappelli F, Bakhordarian A, Thames AD, Du AM, Jan AL, et al. (2015) Ebola: Translational Science Considerations. J Transl Med 13: 11-20.

[18]. Khakshooy A, Chiappelli F (2016) Hypothalamus-Pituitary-Adrenal CellMediated Immunity Regulation in the Immune Restoration Inflammatory Syndrome. Bioinformation 12(1): 28-31.

[19]. Chiappelli F, Iribarren FJ, Prolo P (2006) Salivary biomarkers in psychobiological medicine. Bioinformation 1(8): 331-334.

[20]. Nater UM, Rohleder N, Gaab J, Berger S, Jud A, et al. (2005) Human salivary alpha-amylase reactivity in a psychosocial stress paradigm. Int J Psychophysiol 55(3): 333-342.

[21]. Kirschbaum C, Pirke KM, Hellhammer DH (1993) The 'Trier Social Stress Test'--a tool for investigating psychobiological stress responses in a laboratory setting. Neuropsychobiology 28(1-2): 76-81.

[22]. Kudielka BM, Schommer NC, Hellhammer DH, Kirschbaum C (2004) Acute HPA axis responses, heart rate, and mood changes to psychosocial stress (TSST) in humans at different times of day. Psychoneuroendocrinology 29(8): 983-992.

[23]. Kirschbaum C, Kudielka BM, Gaab J, Schommer NC, Hellhammer DH (1999) Impact of gender, menstrual cycle phase, and oral contraceptives on the activity of the hypothalamus-pituitary-adrenal axis. Psychosom Med 61(2): 154-162.

[24]. Chopra KK, Ravindran A, Kennedy SH, Mackenzie B, Matthews S, et al. (2009) Sex differences in hormonal responses to a social stressor in chronic major depression. Psychoneuroendocrinology 34(8): 1235-1241.

[25]. Jones DA, Rollman GB, Brooke RI (1997) The cortisol response to psychological stress in temporomandibular dysfunction. Pain 72(1-2): 171-182.

[26]. Barkhordarian A, Ajaj R, Ramchandani MH, Demerjian G, Cayabyab R, et al. (2011) Osteoimmunopathology in HIV/AIDS: A Translational EvidenceBased Perspective. Pathol Res Int 2011: 1-13.

[27]. Chiappelli F, Shapshak P, Commins D, Singer E, Minagar A, et al. (2008) Molecular Epigenetics, Chromatin, and NeuroAIDS/HIV: Immunopathological Implications. Bioinformation 3(1): 47-52. 
[28]. Chiappelli F, Manfrini E, Edgerton M, Rosenblum M, Cajulis KD, et al. (2006) Clinical Evidence and Evidence-Based Dental Treatment of Special Populations: Patients with Alzheimer's Disease. J Calif Dent Assoc 34(6): 439-447.

[29]. Chiappelli F, Kutschman MM (2016) Person-Environment (PE) Fit in the Dental Practice. Dental Hypotheses 7(1): 1.

[30]. Barkhordarian A, Demerjian G, Jan A, Sama N, Nguyen M, et al. (2015) Stakeholder Engagement Analysis - A Bioethics Dilemma in Patient-Target- ed Intervention: Patients withTemporomandibular Joint Disorders. J Transl Med 13(1): 15.

[31]. Corstjens PL, Abrams WR, Malamud D (2016) Saliva and viral infections. Periodontol 70(1): 93-110.

[32]. Musso D, Roche C, Nhan TX, Robin E, Teissier A, et al. (2015) Detection of Zika virus in saliva. J Clin Virol 68: 53-55. 